\title{
Lessons Learned from German Research for USAR
}

Q. Hamp, L. Reindl and Alexander Kleiner

\section{Post Print}

N.B.: When citing this work, cite the original article.

C2011 IEEE. Personal use of this material is permitted. However, permission to reprint/republish this material for advertising or promotional purposes or for creating new collective works for resale or redistribution to servers or lists, or to reuse any copyrighted component of this work in other works must be obtained from the IEEE.

Q. Hamp, L. Reindl and Alexander Kleiner, Lessons Learned from German Research for USAR, 2011, IEEE Int. Workshop on Safety, Security and Rescue Robotics (SSRR).

Postprint available at: Linköping University Electronic Press http://urn.kb.se/resolve?urn=urn:nbn:se:liu:diva-72518 


\title{
Lessons Learned from German Research for USAR
}

\author{
Quirin Hamp and Leonhard Reindl \\ University of Freiburg - IMTEK \\ Department of Microsystems Engineering \\ Laboratory for Electrical Instrumentation \\ 79110 Freiburg, Germany \\ Tel: +49 761/2037158 \\ Email: quirin.hamp@imtek.uni-freiburg.de
}

\author{
Alexander Kleiner \\ University of Freiburg \\ Department of Computer Science \\ 79110 Freiburg, Germany
}

\begin{abstract}
We present lessons learned in USAR research within the framework of the German research project I-LOV. After three years of development first field tests have been carried out by professionals such as the Rapid Deployment Unit for Salvage Operations Abroad (SEEBA). We present results from evaluating search teams in simulated USAR scenarios equipped with newly developed technical search means and digital data input terminals developed in the ILOV project. In particular, the "bioradar", a ground-penetrating radar system for the detection of humanoid movements, a semi-active video probe for rubble pile exploration of more than $10 \mathrm{~m}$ length, and the decision support system FRIEDAA were evaluated and compared with conventional search methods. Results of this evaluation indicate that the developed technologies foster advantages in USAR, which are discussed in this paper.
\end{abstract}

Keywords: USAR, information management, technical search, radar, endoscope, emergency communications.

\section{INTRODUCTION}

Key challenges during USAR missions are to quickly localize victims and to efficiently manage the information exchange between responders in the field and the emergency operation center (EOC). I-LOV stands for "Intelligentes, sicherndes Lokalisierungssystem für die Rettung und Bergung Verschütteter" which means when translated "intelligent, securing locating system for the rescue and extraction of trapped victims". The project aims on both the improvement of technical search capabilities, and the improvement of information management during USAR missions. The technologies of the project have been developed in collaboration with the German Federal Agency of Technical Relief (THW). Within this large integration project, novel technical search tools are developed, which are, a robotic platform (Fig. 1(a)) carrying a modular sensor suite [8], a video camera mounted on a stiffening hose, a system for localization of GSM phones (Fig. 1(b)) within a cell [18], and a radar system for detection of humanoid characteristic movements such as breathing (so called "bioradar") which has been employed during the Haiti earthquake response operations in 2010 [14]. Furthermore, a system for personal localization capable of tracking movements within buildings [17], a warning system for imminent structural collapse, and an IT-system for information fusion have been proposed [4].

The key to efficient operations is not only the gathering of accurate information, but also adequate presentation and

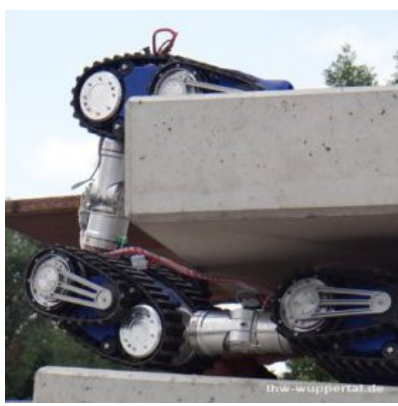

(a) USAR Robot Moebhiu² ${ }^{2}$.

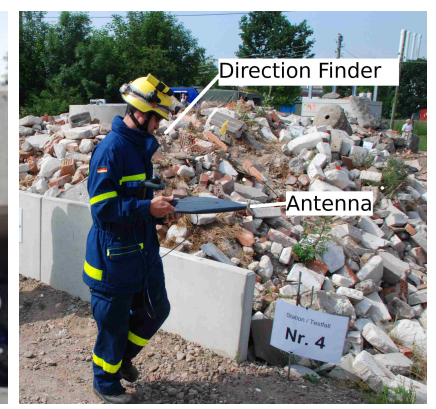

(b) GSM phone localization.
Fig. 1. Search technologies developed in the framework of the I-LOV project.

dissemination. Especially during USAR operations, the collection and presentation of uncertain information from various sources is challenging. Therefore, a Decision Support System called FRIEDAA ${ }^{1}$ has been developed that additionally has the capability to fuse uncertain information about positive and negative results of search activities. In situations where victims are incapable of alerting emergency responders (e.g. due to unconscious), and trapped behind insurmountable obstacles, the mostly utilized method nowadays is to deploy search dogs that might detect body odor. As an alternative, the newly developed bioradar offers a practical search technology which has equivalent capabilities as biologic search, e.g., the detection of alive, trapped, and unconscious victims. In situations where victims are trapped behind obstacles with small open channels, emergency workforces typically employ endoscopic devices to verify the presence of victims and to evaluate their rescue. Unfortunately, current endoscopic devices are limited in their capabilities to penetrate debris, and their video output is difficult to interpret due to missing orientation information [3]. Therefore, a video probe has been developed that tackles both of these issues.

The goal of this paper is to report lessons learned from field tests where these novel approaches for victim localization and information fusion have been deployed. The evaluation was performed by professional USAR workforces of the THW

\footnotetext{
${ }^{1}$ Short for "Functional Remote Information Exchanger with Developing Aggregation Algorithms" IT-system
} 
and in particular of the Rapid Deployment Unit for Salvage Operations Abroad (SEEBA) which is trained for assistance in international disaster response. The assistive search technologies all contributed to more effective search. The synergy with biologic search proved to give more accurate results with respect to certainty, location accuracy, and situation awareness. The assistive IT-system enabling digital communication of messages proved to be a valuable tool and enhance the capability of an EOC to handle messages from the field. However, the chosen communication infrastructure caused insufficient coverage fostering delays in message transmission.

The reminder of this paper is structured as follows. In Section II state-of-the-art protocols of USAR operations are discussed. In Section III the technical tools developed in ILOV are introduced and experiments for testing their deployment by professional response personnel are presented in Section IV. Finally, achieved results and lessons learned are discussed in Section V.

\section{State Of the ART OF USAR Operations}

USAR research in Germany was firstly initiated by Maack during WWII [9]-[11]. He suggested the five phase strategy procedure for finding victims, which aims to ensure high efficiency while decreasing risks for rescuers and trapped survivors. This procedure is nowadays still used by German rescue organizations during USAR missions [16], but has another order than the prioritized one during the USAR operations after the WTC collapse (see [3] p. 380). After first actions (phase I) and the rescue of "surfaced victims", i.e., victims that are easily accessible (phase II), the search for trapped victims is initiated (phase III). If canine search is available, it is the first method of choice. Two to three dogs are conventionally employed. The second dog validates positive search results of the first one. The third dog is used if the other two dogs are exhausted or in order to check after the extrication of a victim whether another one has been forgotten. If the results of the canine search are uncertain, for example when both dogs showed different reactions at the search site, technical search means (if available) are used to verify the results of the dogs. Standard equipment of the THW are acoustic devices. During phase IV trapped victims are extricated. Only after the exhaustive search has been concluded, the area is cleared for systematic removal of heavy debris (phase V).

Operational progress monitoring is commonly based on radio communications or personal messengers. The information is logged in chronological order and highlighted on a tactical map which usually is based either on an aerial image or on a schematic sketch such as presented in Fig. 2. Information categories are differentiated through tactical symbols which are defined in guideline DV1-102 [2].

\section{EVAluated TECHNOLOGIES OF THE I-LOV PROJECT}

Three components of the I-LOV project were evaluated during the field tests: the bioradar system, the video probe,

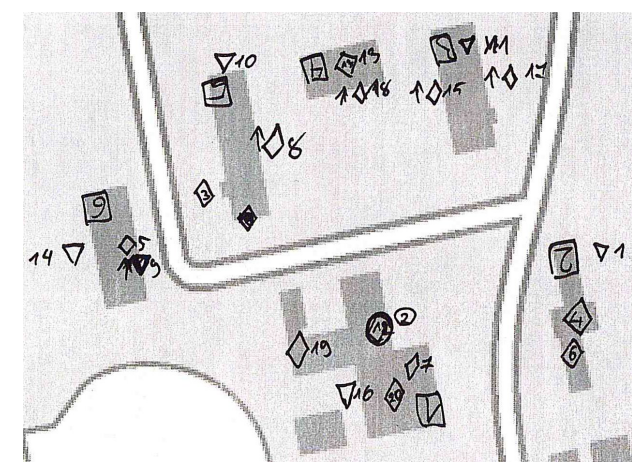

Fig. 2. Conventional map of operational progress during a field test (see Sect. IV-A, p. 3) in 2011 Holzwickede, Germany.

and the IT-System FRIEDAA ${ }^{2}$. These components will briefly be described in the following.

\section{A. Bioradar}

The "bioradar" system is a ground-penetrating radar (GPR) system which enables the detection of repetitive movements such as the heart beat and the chest movement during respiration [14]. Therefore, the bioradar is capable of detecting the presence of either conscious or unconscious survivors situated behind obstacles. The bioradar is a device which needs to rest immobile during an approximatively $30 \mathrm{~s}$ long scan in order to detect human movements of $0.3-4 \mathrm{~cm}$ amplitude. The device is portable by rescue personnel $(\sim 6 \mathrm{~kg})$ and has to be placed above the estimated position of the trapped victim as illustrated in Fig. 6(d), p. 4. The system is composed of antenna, energy supply unit, and integrated circuit board implementing the high-frequency part. The control unit is implemented by a laptop suitable for field use. The laptop is also utilized for the signal processing of the digital raw signal data processed by the circuit board.

\section{B. Semi-active Video Probe}

Passive endoscopic devices such as the telescopic steerable cameras or fiber-optical devices are frequently used for USAR. However, one drawback of this technology is the lack of penetration capabilities within constrained environments such as dense rubble piles. A consequence is that their penetration depth is very limited, usually below a couple of meters. New developments such as the active scope camera of $4 \mathrm{~m}$ length presented by Hatazaki et al. bypasses these limitations [5]. However, another limitation of this device is the user interface, which does not provide any information on position and orientation of the head. This can cause the user to easily loose orientation and thus impede to localize observations within the rubble pile. This has been identified by Casper et al. as major disadvantage during robotic USAR at Ground Zero after the WTC collapse [3].

Therefore, a semi-active, waterproof video probe has been developed in the scope of the I-LOV project that solves these

\footnotetext{
${ }^{2}$ short for "Functional Remote Information Exchanger with Developing Aggregation Algorithms"
} 
issues. It consists of the following three main components: The first component is a hose of variable length $(5-30 \mathrm{~m} \times$ $\varnothing 65 \mathrm{~mm}, \sim 1 \mathrm{~kg} / \mathrm{m}$ ) that can be controlled in its stiffness through air pressure (max. 8 bar ) (see Fig. 3). The video probe can be pushed using this hose up to $10 \mathrm{~m}$ into the rubble pile by a single human user from outside. The second component (600 $\mathrm{mm} \times \varnothing 65 \mathrm{~mm}, \sim 3.4 \mathrm{~kg}$ ) allows the positioning of the head component carrying the video camera through pitch and yaw. The video camera and LED illumination in the head component $(300 \mathrm{~mm} \times \varnothing 80 \mathrm{~mm}, \sim 2.5 \mathrm{~kg}$ ) can be oriented trough two additional degrees of freedom (roll and yaw). The steering of the probe during insertion is performed through the three joints and the so-called "Lindauer Schere", which enables the probe to contrive in a bifurcating propagation channel by ejecting and retracting a structure at the distal part of the head. Sensors for artificial horizons are placed in the second component and in the head, respectively. By this, enhanced situational awareness is offered to the user, for example, when penetrating dense rubble piles, which also enables the localization of observations during the search.

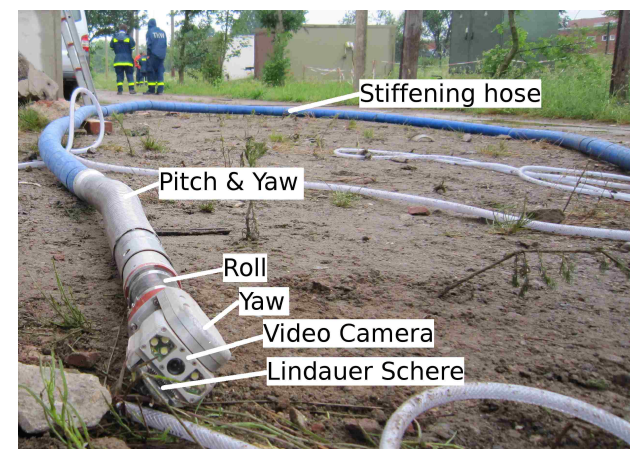

Fig. 3. Semi-active video probe that can travel up to $10 m$ rubble.

\section{Decision Support System FRIEDAA}

The advantages of the use of so called geographic information systems (GIS) have already been recognized and used in projects such as the Japanese DDT project [1]. The collection of relevant information by collaborating operational units and the centralized storage assures enhanced situational awareness. However, GIS typically are limited to store information from reliable observation sources only, whereas in the context of USAR uncertain observations are likely to occur. FRIEDAA is an extension to state-of-the-art GIS that deals with observation uncertainty. FRIEDAA enables rescue workforces, for example, to collect and evaluate uncertain information about victim whereabouts. Information from eye-witnesses and uncertain and imprecise results of search efforts can be collected and attached with geo-reference and timestamp similar to the Kiwi+ format presented by Meguro et al. [12]. For on-site emergency workforces, information collection is facilitated through a PDA application called GeoRescue that allows user inputs by a pointing stick (Fig. 4(b)). The acquisition of a georeference is performed automatically through a GPS receiver. To circumvent the known lacking precision of GPS (see [6]), relative input can be performed or corrected through drag and drop of tactical symbols in the desktop application of FRIEDAA. Furthermore, it enables " $2 \mathrm{~d}+1$ " visualization of horizontal planes in order to track the operational progress in semi-collapsed, multi-storied edifices.

Three main functions assist the SAR team during decisionmaking. First, there is the handling of the victim list which allows for a comparison of victims that were already found and victims that are still expected to be found. Second, for likely victims several search methods can be employed that are all generating results. Multi-level information fusion ${ }^{3}$ allows to associate reports, e.g., from eye-witnesses, that concern the same victim. Furthermore, a victim detection algorithm asses all associated information and finally infers an estimated target location. Third, the positions of rescue forces are assessed automatically, e.g., whether they are within hazardous zones. In specific cases an alert is triggered.

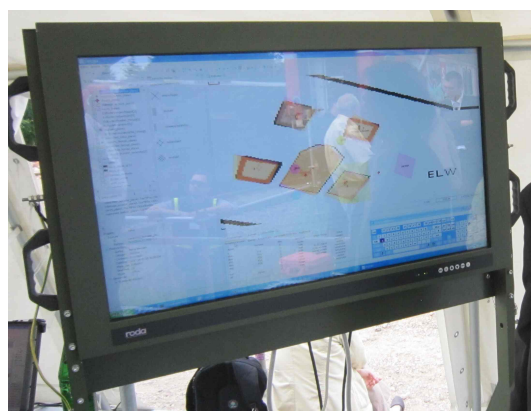

(a) FRIEDAA on 46 inch tactile screen.

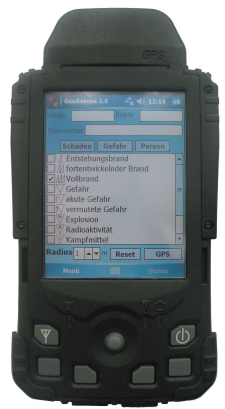

(b) GeoRescue on a PDA.
Fig. 4. Decision Support System FRIEDAA for USAR operations during a field test in 2011 at the German Federal School of the THW in Hoya, Germany.

\section{FIELD TEST RESUlts}

Two field tests have been carried out by SAR professionals to evaluate the proposed methods of the I-LOV project. The first field test focused on the benefits of FRIEDAA during the response to large scale incidents. Especially, communication performance was assessed. The second field test allowed to evaluate the new search technologies. In order to facilitate objective comparisons, both experiments were carried out within two runs, on the one hand with assistive tools of the I-LOV project, and on the other hand without.

\section{A. Evaluation of FRIEDAA for large-scale disasters}

The first field test was carried out in an intact environment of approximatively $3.7 \mathrm{ha}$ that was explored in parallel by four Search and Rescue (SAR) teams (two persons, one team assisted by search dogs) in Holwickede, Germany. In addition to the outside surface, there were six buildings that had as well to be explored indoors (see Fig. 2 for a tactical map). Four of the edifices had two floors. The aim was to find 23 cards (three with uncertain information), and three human victims

\footnotetext{
${ }^{3}$ refer to Nakamura et al. for the definition [13].
} 


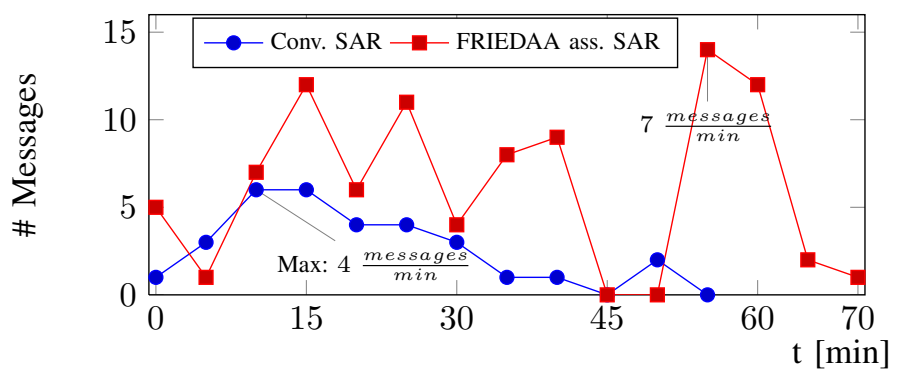

Fig. 5. Comparison of the message volume treated by the EOC between the first and the second run with respect to the duration (t) of the search. The amount of messages that can be managed assisted by FRIEDAA is higher than for conventional SAR.

which were mainly hidden within the buildings. Half of the cards were concerning hazards, the other half victims.

In particular, the EOC was under observation. The first run employed a conventional radio communication infrastructure, the second one was additionally assisted by the FRIEDAA communication infrastructure based on commercial WLAN. During the first run, two SAR operation leaders were in the EOC and managed the operational progress. One was logging all incoming messages chronologically, the other person was busy with plotting information on the map presented in Fig. 2, and with updating a table for better presentation of the information.

The second run employed the same setting, but the teams were assigned to regions they had not explored in the previous run. All search teams carried a PDA with them that they used preferentially to collect and transmit information to the EOC using the GeoRescue application.

During the first run 31 radio messages were transfered in 52 minutes as presented in Fig. 5. The two persons in the EOC had difficulties to handle this amount of incoming messages, which resulted in delays of approximately 5 minutes. The maximal information flow treatable by the EOC was about four messages per minute. A comparison of the map in Fig. 2 with the actual situation resulted in the fact that during the first run two victim observations and three hazard observations were missed. Furthermore, the locations of the observations were only approximative and did not correspond to the actual positions since emergency workforces only indicated the geographic direction and the floor.

The second run lasted in total 69 minutes during which 57 messages were received in the EOC. Considering the fact that the GeoRescue application allows to transfer messages containing multiple data (e.g. a hazard combined with information about a person), and that during the previous run every single radio communication message was accounted for, a total of 92 successfully transmitted messages has to be considered. Furthermore, every time an information was collected at the EOC, the region around the acquired georeference was automatically marked as cleared by FRIEDAA, which increased the amount of messages to 149 in total. In Fig. 5 messages about cleared zones are not accounted for, because they were automatically sent. The identification of the search team was as well transferred automatically to the FRIEDAA system.

Since the WLAN network did not cover the whole area, messages were sent delayed, e.g., once a position within communication range has been reached again after loosing connection. Furthermore, the lacking GPS reception indoors constrained the SAR team members to locate themselves near windows to receive GPS signal. They had to indicate whether the message concerned the first or the second floor. The search performance was better in the second run than in the first run, only one hazard was missed. A comparison of the performance of both runs is presented in Table I.

TABLE I

PERFORMANCE OF THE TWO RUNS EVALUATING FRIEDAA FOR LARGE SCALE DISASTERS.

\begin{tabular}{|l||l|l|l|l|}
\hline RUN & $\begin{array}{l}\# \\
\text { INFOS } \\
\text { MISSED }\end{array}$ & $\begin{array}{l}\text { AVG. } \\
\text { MESSAGES } \\
{\left[\mathrm{min}^{-1}\right]}\end{array}$ & $\begin{array}{l}\text { AVG. } \\
\text { DELAY } \\
{\left[\mathrm{min}^{-1}\right.}\end{array}$ & $\begin{array}{l}\text { TOTAL } \\
\text { SEARCH } \\
\text { TIME }[\mathrm{min}]\end{array}$ \\
\hline \hline CONVENTIONAL & 5 & 0.60 & n.a. & 52 \\
\hline FRIEDAA & 1 & 2.16 & 8 & 69 \\
\hline
\end{tabular}

\section{B. Evaluation of assistive search technologies integrated in conventional USAR procedure}

The second field test took place in a completely destroyed area of $0.58 \mathrm{ha}$ with ten rubble piles at the school of the THW in Hoya, Germany. Three human victim actors were hidden in tunnels under the rubble piles. Furthermore, a worn piece of cloth was placed within one rubble pile, in order to check whether the dogs would neglect correctly this false target. As in the first field test, two runs were performed, but FRIEDAA assisted in both of the cases.

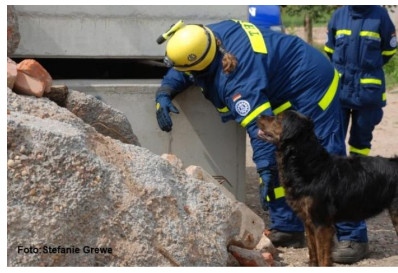

(a) Biologic Search.

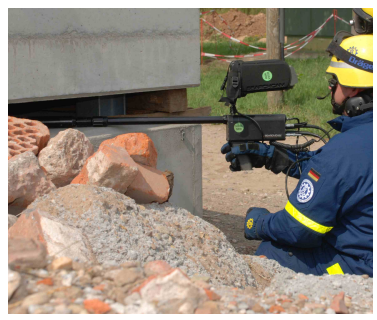

(c) SearchCam.

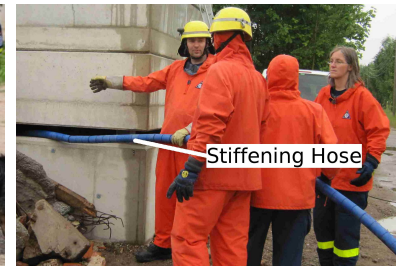

(b) Semi-Active Video Probe.

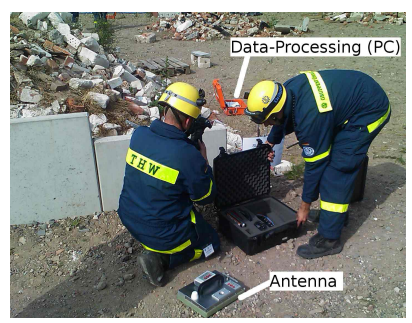

(d) Ground-penetrating Bioradar.
Fig. 6. Synergy between biologic and technical search methods.

During the first run only biologic search was performed after a exploration phase. There were two exploration teams who covered the whole area during 16 minutes. Two search 
dog teams were employed to search the whole area. After 27 minutes all victims were found. In one situation a direct access to the victim could not be achieved. The search team suggested the application of technical search methods which, however, were not allowed during the first run. The total duration of the first run was 43 minutes with 93 distinct messages.

The second run was managed by the SEEBA team members. The same scenario as in the first run was used, but assistive technical search assets were available such as the bioradar, a SearchCam 3000, and the semi-active video-endoscope. The total duration of the exploration phase also performed by two teams was 25 minutes. The search of two search dog teams lasted 28 minutes. The search dogs indicated correctly all locations of the victims. However, since technical search assets were available, they were used to verify the positions where dogs had indicated a victim. One victim that was trapped under stacked concrete slabs could be located thanks to the videoprobe (Fig. 6(b)). In this case, biologic search (Fig. 6(a)) and the SearchCam (Fig. 6(c)) would have had limited success. The other two victims were both verified successfully with the bioradar. After extrication of one victim, the bioradar device was employed once again to clear the area. The situation after the search phase gathered in FRIEDAA is presented in Fig. 7. The duration of the second run was 77 minutes with a total of 54 messages.

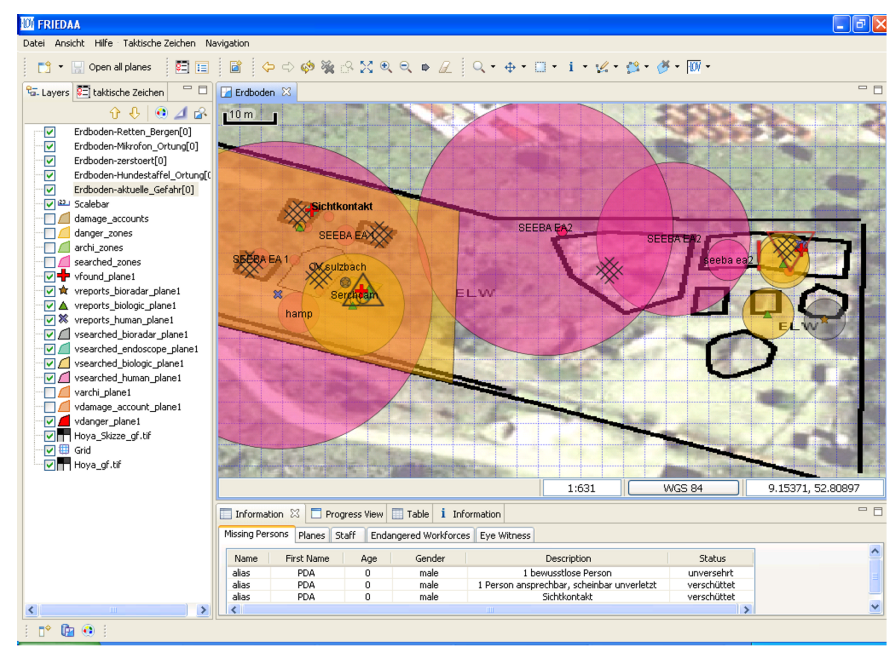

Fig. 7. Representation of the operational progress in FRIEDAA after the field test with assistive search technology. At the bottom, there is the list of the affected persons. Left is the control of the layers and central the geographical representation of the situation at the end of the search phase.

The operational progress of both runs is presented in Fig. 8 . It shows that the longer total duration of the second run is mainly due to the employment of technical search means. The difference in message volume is due to the fact that during the first run, more hypothetic hazards $(+15)$, more destruction zones $(+29)$, and less details about person $(-8)$ were collected than in the second run. A comparison of the performance of the two runs is presented in Table II.

The performance of search activities can be expressed in how fast an area is cleared, i.e. the spatial search performance.

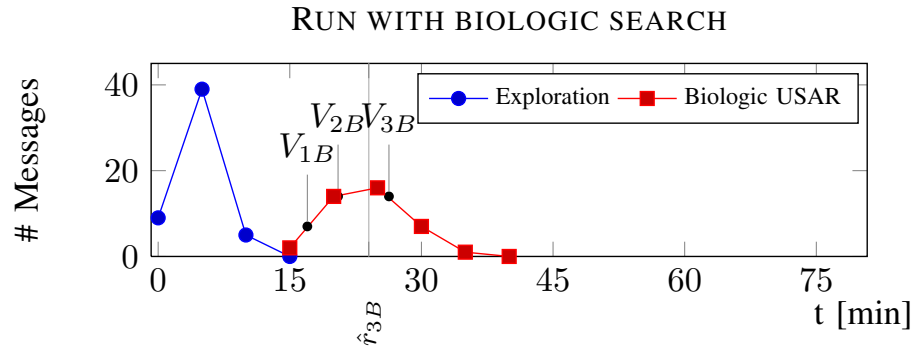

RUN WITH ASSISTIVE TECHNOLOGIES

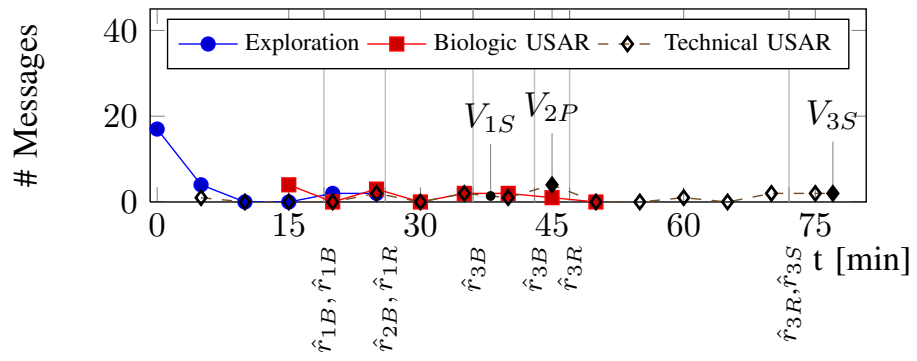

Fig. 8. Operational progress of the USAR operations with three trapped victims with respect to the messages of phases: Exploration, Biologic USAR and Technical USAR. The timestamps of all collected reports $\left(\hat{r}_{i x}\right)$ and victims $\left(V_{i x}\right)$ are represented relative to each victim $(\mathrm{i}=1,2,3)$ and employed method ( $\mathrm{x}=$ SearchCam (S), Video Probe (P), Biologic Search (B), Bioradar (R))

TABLE II

PERFORMANCE OF THE TWO RUNS EVALUATING THE ASSISTIVE SEARCH TECHNOLOGIES WITH FRIEDAA ASSISTANCE.

\begin{tabular}{|c|c|c|c|c|}
\hline RUN & $\begin{array}{l}\text { AVG. } \\
\text { RE- } \\
\text { PORTS / } \\
\text { VICTIM }\end{array}$ & $\begin{array}{l}\text { AVG. TIME } \\
\text { FIRST DE- } \\
\text { TECTION } \\
{[\mathrm{min}]}\end{array}$ & $\begin{array}{l}\text { AVG. } \\
\text { MESSAGES } \\
{\left[\mathrm{min}^{-1}\right]}\end{array}$ & $\begin{array}{l}\text { TOTAL } \\
\text { SEARCH } \\
\text { TIME } \\
{[\text { min }]}\end{array}$ \\
\hline CONVENTIONAL & 1.3 & 21.7 & 2.16 & 43 \\
\hline $\begin{array}{l}\text { ASSISTIVE } \\
\text { SEARCH TECH. }\end{array}$ & 4.0 & 24.0 & 0.69 & 77 \\
\hline
\end{tabular}

The performance for human exploration and biologic search in a heavily destroyed area is $1.4 \mathrm{ha} / \mathrm{h}$ and $1.1 \mathrm{ha} / \mathrm{h}$, respectively. These numbers are the mean over both runs of the second field test. It is worth noting that the spatial search performance of the biologic search method consists of covering the whole area twice with two different dogs.

\section{Discussions \& LESSONS LEARNED}

In Germany, USAR compared to surface search of lost persons only represents $5 \%$ of all SAR operations (statement of a canine SAR team of the THW). The SAR team members appreciated particularly the capability of FRIEDAA to show the cleared surfaces. They stressed that FRIEDAA would increase the surface search efficiency. The presented field tests partially allowed to evaluate the efficiency enhancement of the assistive technology of the I-LOV components. Since the extrication phase was not part of either of the field tests, the advantages of more accurate localization through assistive technologies could not be demonstrated quantitatively. 
Despite the fact that commercially available GPS receivers are insufficiently precise for victim localization, and also not acquirable indoors, the capability to store information with timestamps and geo-reference turned out to be very promising. Ongoing developments about Pedestrian Dead Reckoning (PDR) [17] and SLAM [7] will allow robust localization in all circumstances and enhance the capability to log operational progress in complex three dimensional terrain.

Conventional maps of operational progress such as presented in Fig. 2 are poor in content and functionality compared to GIS supported maps such as delivered by FRIEDAA (see Fig. 7). Long lasting operations in complex, multi-storied terrain are certainly manageable on paper media. However, the capability to search and filter information with respect to temporal timestamps and categories are the clear advantages of digital assisted search. The data logging capability of digitally assisted search fosters the consolidation of experiences in the field, which is important for improving the efficiency of USAR missions in the future.

The benefits of non-verbal digital transmission of messages became evident through the first field test. In the first run with conventional methods, the two persons in the EOC reached their limit to handle the amount of incoming messages. In the second run, more information was transmitted whereas the two persons in the EOC had nearly no work and would have been able to focus on other important tasks such as warning endangered workforces, assessing uncertain information and managing the list of missing persons. The WLAN communication infrastructure was lacking of coverage within and behind buildings during the first field test. The lack of coverage caused delays. However, this can be remedied with other digital radio communication technology such as GSM or TETRA [15]. If such an infrastructure is used to entirely cover a terrain such as during the second field test, delays become negligible.

The comparison in Fig. 8 shows that the assistive technology used during the second run increased the total duration of the search phase. However, it is worth noting that during USAR missions precise victim localization is of top most importance, which can be gained by assistive technologies as proved by the performance comparison in Table II. If the position is only known vaguely, extrication efforts and the total rescue duration are considerably increasing. The SEEBA stressed the importance of portability of the assistive technologies. The bioradar was particularly promising in this regard. The assistive search technologies have shown to be trustful tools that increase certitude about victim presence and location. The search technologies in the given circumstances of the field test in Hoya always indicated correctly the presence of a trapped victim.

\section{ACKNOWLEDGMENT}

We gratefully acknowledge financial support from the German Federal Ministry of Education and Research (support code: 13N9759). Furthermore, we are most grateful for the support of the German Federal Agency for Technical Relief (THW), of SEEBA, of the companies JT-electronic GmbH and Berlin- Oberspree Sondermaschinenbau GmbH (BOS). FRIEDAA uses uDig source code of Refractions Research.

\section{REFERENCES}

[1] Hajime Asama, Yasushi Hada, Kuniaki Kawabata, Itsuki Noda, Osamu Takizawa, Junichi Meguro, Kiichiro Ishikawa, Takumi Hashizume, Tomowo Ohga, Michinori Hatayama, Fumitoshi Matsuno, and Satoshi Todokoro. Rescue infrastructure for global information collection. In 2006 SICE-ICASE International Joint Conference, Vols 1-13, pages 1600-1605, 2006.

[2] Bundesanstalt Technisches Hilfswerk, Deutschherrenstraße 93-95, 53177 Bonn. THW DV1-102 Taktische Zeichen, 2000.

[3] Jennifer Casper and Robin Murphy. Human-Robot Interactions During the Robot-Assisted Urban Search and Rescue Response at the World Trade Center. IEEE Transactions on Systems, Man, and CyberneticsPart B: Cybernetics, 33(3):367-385, 2003.

[4] Quirin Hamp, Michael Eitelberg, Bo-Sik Lee, Tobias Becker, Dirk Wiebeck, and Leonhard Reindl. Information Fusion based on graph analysis during Urban Search and Rescue. In Information Fusion, Edinburgh, UK, 2010. IET.

[5] K. Hatazaki, M. Konyo, K. Isaki, S. Tadokoro, and F. Takemura. Active scope camera for urban search and rescue. In Intelligent Robots and Systems, 2007. IROS 2007. IEEE/RSJ International Conference on, pages 2596 -2602, 29 2007-nov. 22007.

[6] J. Hightower and G. Borriello. Location systems for ubiquitous computing. Computer, 34(8):57 -66, 2001

[7] Alexander Kleiner, Christian Dornhege, and Sun Dali. Mapping disaster areas jointly: RFID-coordinated SLAM by humans and robots. In International Workshop on Safety, Security and Rescue Robotics, pages 42-47, NEW YORK, USA, 2007. IEEE.

[8] Patrick Labenda, Tim Sadek, and Thomas Predki. Controlled maneuverability of an articulated tracked mobile robot. ASME Conference Proceedings, 2010(44106):1141-1147, 2010.

[9] P. Maack. Die Systematik der Schadenstellen, I. Teil. Baulicher Luftschutz, 6(5):97-118, 051942.

[10] P. Maack. Die Systematik der Schadenstellen, II. Teil. Baulicher Luftschutz, 6(6):129-133, 061942.

[11] P. Maack. Die Systematik der Schadenstellen, III. Teil. Baulicher Luftschutz, 7:2-16, 1943.

[12] Jun-Ichi Meguro, Kiitirou Ishikawa, Takumi Hasizume, Jun-Ichi Takiguchi, Itsuki Noda, and Mitinori Hatayama. Disaster information collection into geographic information system using rescue robots. In 2006 IEEE/RSJ International Conference on Intelligent Robots and Systems, volume 1-12, pages 3514-3520, 2006.

[13] Eduardo F. Nakamura, Antonio A. F. Loureiro, and Alejandro C. Frery. Information fusion for wireless sensor networks: Methods, models, and classifications. ACM Computing Surveys, 39(3), 2007.

[14] O. Rogall, R. Schilling, M. Loschonsky, R. Schimko, and L. M. Reindl. Radargestützte Personenortung. In Sensoren und Messsysteme, pages 728-732, Nürnberg, 2010. VDE. (engl.: Radar based localization of persons).

[15] Peter Stavroulakis. Terrestrial trunked radio - TETRA: a global security tool. Springer, 2007.

[16] Ulf Tabbert and Jörg Meike. Maßnahmen der Feuerwehr und anderer Hilfskräfte nach Gebäudeeinstürzen. Vereinigung zur Förderung des Deutschen Brandschutzes (vfdb), 2003.

[17] R. Zhang and L.M. Reindl. Pedestrian motion based inertial sensor fusion by a modified complementary separate bias Kalman filter. In Sensors Applications Symposium (SAS), pages 209-213. IEEE, 2011.

[18] S. Zorn, M. Maser, A. Goetz, R. Rose, and R. Weigel. A power saving jamming system for E-GSM900 and DCS1800 cellular phone networks for search and rescue applications. In IEEE Topical Conference on Wireless Sensors and Sensor Networks (WiSNet), 2011. 